\title{
E-healthcare via Customized Information Services: Addressing the Need for Factually Consistent Information
}

\author{
Syed Sibte Raza Abidi ${ }^{1}$ and Yong Han Chong ${ }^{2}$ \\ ${ }^{1}$ Faculty of Computer Science, Dalhousie University, Halifax B3H 1W5, Canada \\ sraza@cs.dal.ca \\ ${ }^{2}$ School of Computer Sciences, Universiti Sains Malaysia, Penang 11800, Malaysia \\ thestepdcs.usm.my
}

\begin{abstract}
Web portals provide an efficient gateway to a broad range of Eservices, resources and information. Web portals need to evolve towards being adaptive in nature, so that the ensuing E-services provided by them are dynamically tailored to meet the diverse needs of its users. This paper explores the use of intelligent techniques, in particular constraint satisfaction methods, to develop adaptive E-services that provide customized and factually consistent information to users. We model the generation of customized information content as a constraint satisfaction problem-a solution is derived by (a) satisfying user-model constraints with information document selection constraints; and (b) establishing inter-document consistency when dynamically combining heterogeneous information documents. The work is applied in an E-Healthcare setting leading to the generation of personalized healthcare information.
\end{abstract}

\section{Introduction}

Web portals provide an efficient gateway to a broad range of open, remote and ubiquitous electronic services, resources and information-the so-called E-Services portfolio $[1,2]$. The emergence of vertical portals-i.e. portals offering services and content aimed for a specific community-such as Enterprise information portals, Knowledge portals and Internet portals allow access to enterprise-specific information/services for an audience that have may have different goals, interests, preferences, intellectual levels and consumption capacity $[3,4]$.

A web portal interacts with a broad-base of heterogeneous users. Hence, a one size fits all model for web content and service design does not necessarily satisfy the entire spectrum of user needs/goals. Given that it is impossible to develop an exclusive web portal for each user class, one solution is to develop adaptive web portals-i.e. develop a base-set of both generic E-services and information content that can be dynamically tailored to meet the diverse goals/needs of the users [5]. This brings to relief an interesting constraint satisfaction problem, whereby the problem space on the one hand encompasses a wide diversity of E-service/information needs of the 
users, and on the other hand a profusion of generic E-services/information content. Our novel constraint satisfaction based solution therefore involves matching the specific user needs/goals with the most effective and appropriate E-service/information content. Our IC approach goes even further; we argue that the customization of both E-services and information content should not only be limited to satisfying the user profile, rather it is important that any dynamically customized (a) E-service should satisfy all business rules; and (b) information content should be factually and contextually consistent-i.e. no aspect of the customized information content should appear to be in contradiction with any other information simultaneously presented to the user. Hence constraint satisfaction methods need to be applied at two levels: (1) satisfying the user-profile constraints when selecting from generic Eservices/information content; and (2) satisfying the factual consistency between the selected information content in order to ensure that the cumulative information content is factually consistent.

This paper explores the use of intelligent techniques, in particular constraint satisfaction methods, to develop adaptive E-services that provide customized information services via a web portal. Adaptive E-services and information presentation have been pursued using adaptive hypermedia systems in conjunction with model-based and rule-based methods [6]. In our work, we present a novel approach whereby we model the generation of adaptive and customized information content as a constraint satisfaction problem - a solution is derived by (a) satisfying user-model constraints vs. information/service selection constraints; and (b) establishing information content consistency when combining heterogeneous information objects by satisfying information consistency constraints associated with each selected information object. We argue that, establishing the factual consistency of the information provided to users is an important, yet often neglected, issue. Functionally, it is possible that the combination of two information objects, both relevant to the user model, can inadvertently lead to a contradictory situation-i.e. one recommending whilst the other restricting from an action/decision at the same time. Hence, information customization methods need to check whether two information objects can be amicably synthesized-i.e. they jointly do not lead to any contradictions - to generate personalized information content. We believe that the incorporation of constraint satisfaction based information customization methods within E-services setting can reduce the cognitive overload experienced by E-service users and also improve the usability, acceptance and effectiveness of an interactive vertical portal.

We present an Information Customization (IC) framework that (a) generates personalized information via the dynamic selection and synthesis of multiple topicspecific documents that are deemed relevant to a user profile [7]; (b) satisfies the factual, contextual and functional consistency of the compiled personalized information via inter-document constraint satisfaction; and (c) delivers the personalized information via a vertical portal. A unique hybrid of adaptive hypermedia and Constraint Satisfaction (CS) methods is suggested to realize an IC framework that maximizes collaboration and minimizes conflicts between a set of hypermedia documents whilst generating personalized information. IC is achieved by satisfying user-model constraints and inter-document (factual consistency) constraints using a variety of CS 
methods. This work is applied to an experimental E-Healthcare setting that provides personalized healthcare information in the realm of a patient empowerment programme [8].

The paper is organized as follows: In section 2 we provide an overview of IC in general. In section 3 we briefly introduce constraint satisfaction methods and then lead to our constraint satisfaction based IC methodology. In section 4, we discuss the IC methods that we have developed. In section 5 we present a working example of IC, vis-à-vis personalizing healthcare information, using our IC methodology and methods. Finally, in section 6 we present our conclusions and future outlook.

\section{A Brief Overview of Information Customization}

At the forefront of adaptive services initiatives are adaptive hypermedia systems [9, $10,11,12]$ that provide an umbrella framework incorporating hypermedia, artificial intelligence and web technology to develop and deploy web-based dynamically adaptive E-service systems [5].

IC research offers interesting insights into ways for (a) profiling users based on information about the user's demographic data, knowledge, skills, capabilities, interests, preferences, needs, goals, plans and/or usage behaviour; and (b) pro-actively adapting the content, structure and/or presentation of hypermedia objects, based on the user's profile, to make an ostensive impact on the user. Each of the abovementioned IC modalities involves different methods and strategies, for instance:

- Content adaptation is achieved via page variants [13], fragment variants [14, 7], Adaptive stretchtext [15] and adaptive natural language generation methods [16]. Our work is based on the notion of fragment variants, whereby fragments of texts are authored to cover the entire diversity of the topic, where each text fragment corresponds to a particular user characteristic. At runtime, a set of fragments is amalgamated to realize the final personalized document.

- Structure adaptation involves the dynamic changing of the link structure of hypermedia documents. Collateral structure adaptation [15], link sorting [17], link annotation [18], and link removal/addition [19] are some of the methods used. Our work does not involve any structure adaptation, as this kind of adaptation is relevant to an existing hypermedia document/website.

- Presentation adaptation leads to changes in the layout of the hypermedia document. Typically, the changes involve text positioning (or focusing), graphics and multimedia inclusion/exclusion, background variations and GUI interface. In our work, the use of a presentation template allows for presentation adaptation based on the user's preferences and information browsing behaviour. 


\section{Our Information Customization Operational Framework}

Constraints arise in most areas of human endeavor and we are used to solving them. In a computational sense, constraints mathematically formalize the dependencies in a physical world in terms of a logical relation among several unknowns (or variables), each taking a value from a defined domain. In principle, a constraint restricts the possible values that the variables can take whilst solving a problem. Constraint programming solves problems by stating constraints about the problem area and, consequently, finding solutions that may 'satisfy' all the constraints. A Constraint Satisfaction Problem is defined by a tuple $P=(X, D, C)$ where $X=\left\{X_{1}, \ldots, X_{n}\right\}$ is a finite set of variables, each associated with a domain of discrete values $D=\left\{D_{l}, \ldots, D_{n}\right\}$, and a set of constraints $C=\left\{C_{1}, \ldots, C_{l}\right\}$. Each constraint $C_{i}$ is expressed by a relation $R_{i}$ on some subset of variables. This subset of variables is called the connection of the constraint and denoted by $\operatorname{con}\left(C_{i}\right)$. The relation $R_{i}$ over the connection of a constraint $C_{i}$ is defined by $R_{i} \subseteq D_{i l} \times \ldots \times D_{i k}$ and denotes the tuples that satisfy $C_{i}$. A solution to a constraint satisfaction problem is an assignment of a value from its domain to every variable, in such a way that every constraint is satisfied [20, 21, 22].

\subsection{Information Customization Approach}

Our CS-mediated IC approach specifically caters for the following operational conditions:

- A user profile, comprising a number of user-defining attributes, describes the characteristics of each user.

- IC is achieved using constraint satisfaction methods that involve the satisfaction of two constraints: (i) user-model constraints and (ii) co-existence constraints.

- The information content is organized as a corpus of topic-specific hypermedia documents, where each topic may be described by a number of documents.

- Each document is composed of two sections: (a) Condition section that specifies the conditions for the selection of the document; and (b) Content section that comprises the information content.

- The condition section comprises two types of conditions: (a) document-selection conditions that are compared with the user's profile in order to determine whether the said document is relevant to the user. A document is selected if all documentselection conditions are satisfied; and (b) document-compatibility conditions determine whether the said document can mutually co-exist with other selected documents-i.e. the selected documents do not suggest contradictory information.

- The content section is sub-divided into two segments: (i) unrestricted content segment which is available when the document is selected; and (ii) restricted content segment which has the same structure as the document itself, but is available for limited use, subject to the satisfaction of its associated conditions. 
- The final personalized information package is a systematic collection of userspecific and mutually consistent hypermedia documents that is delivered to endusers using web-based mechanisms.

In line with our IC approach we have developed a Java-based IC system (see Fig. 1) to generate personalized healthcare information. The CS methods and the userinterface for the IC website have been developed in Java. The information content is written as XML documents, and is transformed to HTML to be pushed to the users via email. Also we have developed a dedicated XML-based document editor for medical professionals to author both the information content and associated constraints.

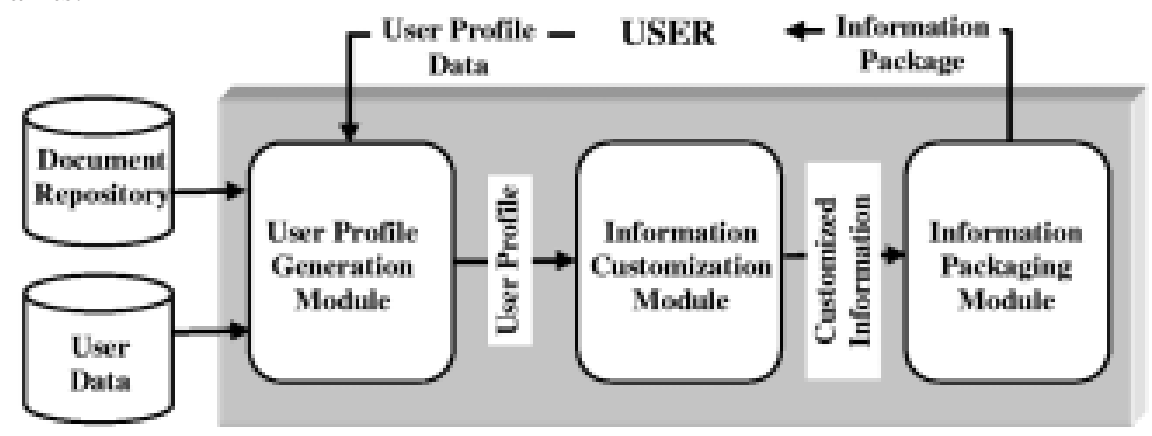

Fig. 1. The architecture of our IC system comprising 3 main modules

\subsection{Operational Pre-requisites for Information Customization}

We would like to point out that at present our IC approach presupposes the existence of the following pre-requisites (which are not addressed by us in this research).

- The content and constraints for each document is a priori specified by domain experts. We do not have a specific knowledge engineering methodology whereby domain experts can specify the content and the constraints for a variety of new topics and enhance existing ones. Hence, at present we work with a much smaller set of documents than required in a real-life setting.

- A complete user-profile is given. Our IC methodology addresses user profiling by acquiring current user data from an online data repository and subsequently verifying it with the user. However, at present the methods to generate the user-profile are not operational.

- The classification of the various topic-specific documents is predicated by a simple yet static domain-specific ontology. We foresee the need to develop means to enable the evolution of the ontology together with the automatic re-classification of the documents. At present, we do not focus on sophisticated methods for document classification, but intend to look into this issue in future research as it impacts IC. 


\section{Information Customization Methodology}

The proposed IC methodology takes into account the above operational considerations and prerequisites, and provides a technical solution towards (a) the satisfaction of user-model constraints to select user-specific information; and (b) the satisfaction of co-existence constraints to ensure that the selected information is factually, functionally and contextually consistent. In principle, the above demands a conflict evaluation framework, hence we leverage variants of CS consistency-checking techniques and CS search algorithms to generate customize information. Below we list the distinct steps of our IC methodology (shown in Fig. 2).

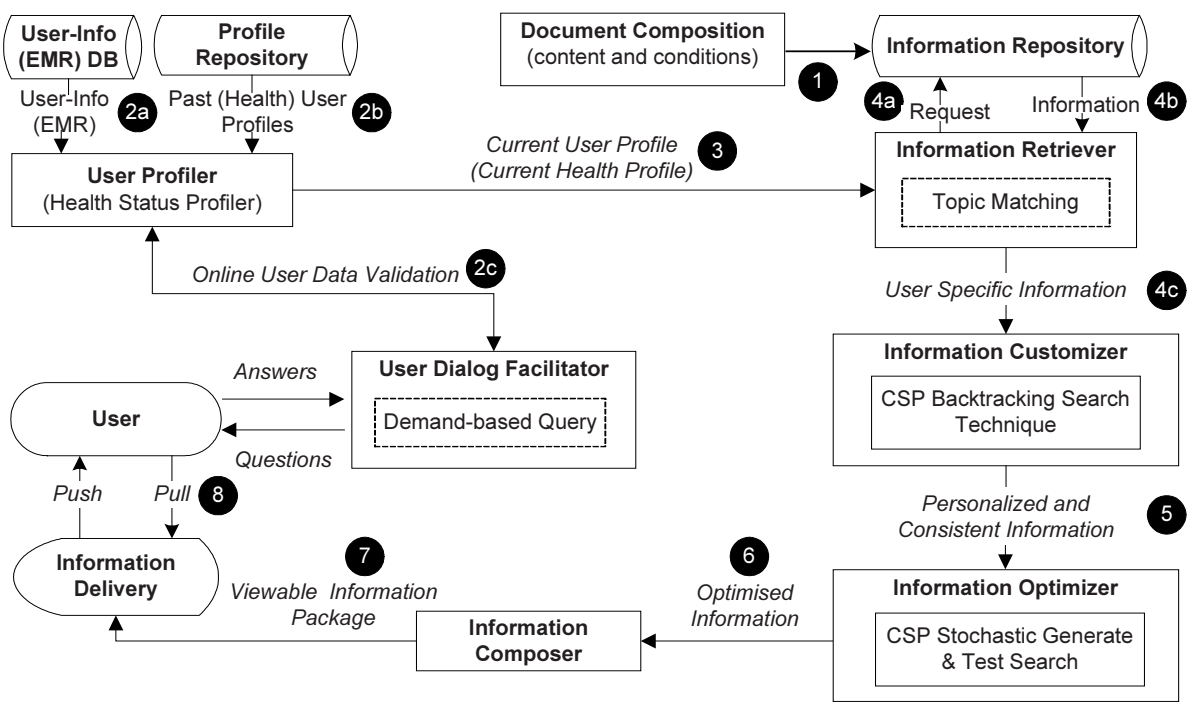

Fig. 2. Process flow for generating personalized and factually consistent information

Our IC methodology is used to generate personalized healthcare information, however we emphasize that the proposed IC methodology is applicable to other information dissemination problems.

1. Document composition: For each topic-specific document, its information content and associated conditions need to be specified by the document authors. We use conditional frame-based representation for representing each document, as it supports our overall information customization strategy.

2. Acquisition of user-profile specific information: User-defining information can be acquired from stored personal records (in our case from user's electronic medical record with the permission of the user), past user-profiles, and if needed directly from the user via a web-based dialogue.

3. Generation of the user profile (i.e. user model): For optimal impact of the disseminated information, the user's profile needs to be current and accurate. Based on the collected user information, we prepare a representative user profile that serves as the basis for information customization. 
4. Selection of user-specific information content: Based on the user-profile, relevant documents are selected from the document corpus. Document selection involves the use of CS techniques to satisfy the document-selection conditions of each document with the user's critical attributes noted in the user-profile. Here, we collect a set of candidate documents that individually are relevant to the user-profile.

5. Selection of factually compatible information content: Given a set of candidate documents, it is important to ensure that they can potentially co-exist without causing any factual inconsistency. We begin by establishing the minimum information coverage that is factually consistent-i.e. establishing the core document set which includes one document for each topic that need to be discussed in the final information package. We use a CS based backtracking search method to globally satisfy the document-compatibility conditions for all the selected documentsany document that is deemed factually inconsistent with the rest of the documents is discarded. The ensuing core document set covers each topic of interest; yet depict the minimum consistent information coverage that can be provided to the user.

6. Maximizing the information coverage: Given the core document set, we next attempt to maximize the information coverage by checking whether it is possible to include any previously non-selected candidate document to the core document set, whilst maintaining overall factual consistency. A CS based stochastic generate-and-test algorithm is used for this purpose. The outcome is the potential maximization of the information coverage (from the baseline determined by the core documents) manifested in terms of an optimized presentation document set, which constitutes the final personalized information content.

7. Preparation of information package: The information content (of the documents) within the presentation document set is in XML format. Depending on the user's viewing medium, the information content (in XML) is transformed to HTML, WML or PDF format to realize the personalized information package.

8. Delivery of information package: Finally, the personalized information package is delivered to the user over the Internet. Two delivery mechanisms are supported: (a) push-based - the information package is pro-actively e-mailed to the user, and (b) pull-based-the user retrieves it from the website.

\section{Information Customization Methods}

\subsection{Information Representation}

Our information representation scheme involves the binding of information content segments with a set of conditions $[10,11,15,23,24]$, in the form of a conditional frame (shown in Fig. 3).

The notion of associating information content with its selection criteria is quite relevant in a healthcare setting where there is a necessity to ensure that any information provided to users is not only consistent with the individual's needs but it also should not inadvertently prove to be harmful, confusing and contradictory in any 
way. Note that, each condition is representation by the tuple (type, value, weight). The weight defines the degree of restriction ranging from 0 to 1 , where $0 \rightarrow$ strictly not recommended and $1 \rightarrow$ highly recommended.

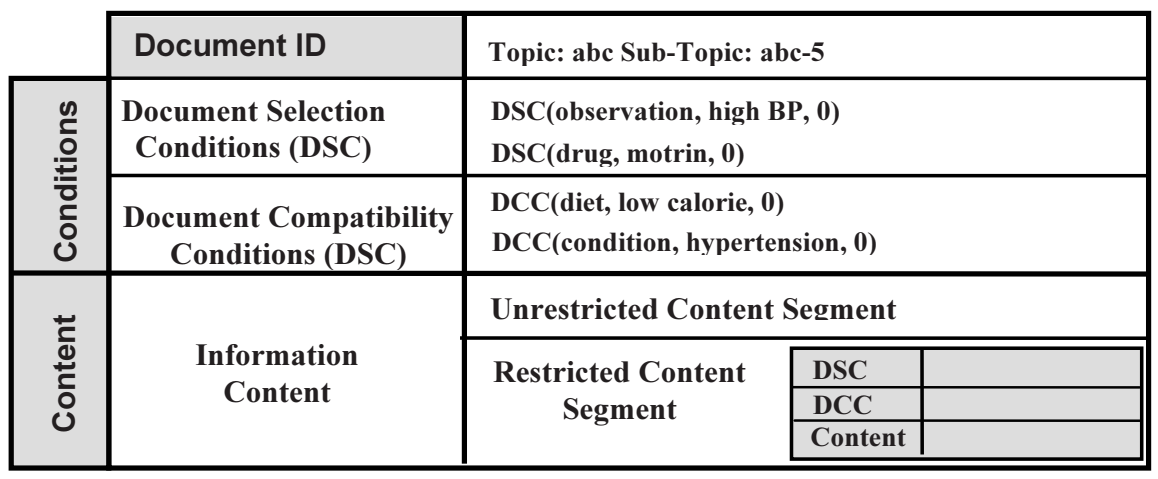

Fig. 3. Architecture of a conditional frame-based hypermedia document for IC

\subsection{Constraint Satisfaction Methods for Information Customization}

After having retrieved topic-specific documents relevant to a given user profile, here we illustrate the modelling of our information customization problem as a CS problem. The specification of a CS problem requires a set of variables, its domain values and constraints.

We define IC via CS problem-solving as (a) a set of information topics, represented in terms of topic-variables $\mathrm{X}=\left\{\mathrm{X}_{1}, \ldots, \mathrm{X}_{\mathrm{n}}\right\}$, where for each topic-variable $\mathrm{X}_{\mathrm{i}}$, there is a finite set of (topic-specific) documents. The set of documents associated with each topic-variable is deemed as its domain, $\mathrm{D}_{\mathrm{i}}$; (b) a user profile represented as a single-valued user-variable; and (c) and two types of constraints-user-model constraint (UMC) and co-existence constraint ( $\mathrm{CoC})$. A solution to our CS problem is the systematic selection of the largest subset of documents for each topic-variable, in such a way that the ensuing user-model and co-existence constraints (amongst all selected documents) are fully satisfied. Such a CS solution can be obtained by searching, via CS search methods, the domain for each topic-variable.

\subsubsection{User-Model Constraint Satisfaction: Generating the Candidate Document Set}

A user-model constraint between a topic-variable and a user-variable is satisfied when all the documents in the domain of the topic-variable are consistent with user profile. The general idea is to compare the document-selection conditions (DSC) for each document with the user critical attributes (UCA) listed in the user profile. This is achieved by calculating the conflict value (CV), as shown below, between the DSC and UCA. A low CV value implies that the user-model constraint is not breached and that the document is seemingly relevant to the user, whereas a high $\mathrm{CV}$ value denotes 
the irrelevance of the document to the user. Typically, a user-defined threshold is used to determine the acceptable level of CV.

$$
\begin{gathered}
c v_{D S C}^{U C A}=\mid(\text { weight })_{D S C}^{D o c}-(\text { weight })_{U C A}^{U P} \mid,(\text { type,value })_{D S C}^{\text {Doc }}=(\text { type, value })_{U C A}^{U P} \\
0 \leq c v_{D S C}^{U C A} \leq 1,0 \rightarrow \text { not breached, } 1 \rightarrow \text { breached }
\end{gathered}
$$

The conflict value for a DSC is the modulus of the difference between the weights of the DSC and the matching UCA. Note that a matching UCA has the same type and value as the DSC in question.

To satisfy the user-model constraint we employ a variation of CSP nodeconsistency technique-the recursive-level node-consistency algorithm [25]. The working of our modified recursive-level node-consistency algorithm is as follows: for each topic-variable, if the domain contains a document that is inconsistent to user profile, then that particular document is removed from the domain. Eventually, only documents that are consistent to user profile-i.e. relevant to user-are retained in each topic-variable's domain and the resulting set of user-specific documents are regarded as the candidate document set.

\section{Algorithm Recursive-level NC}

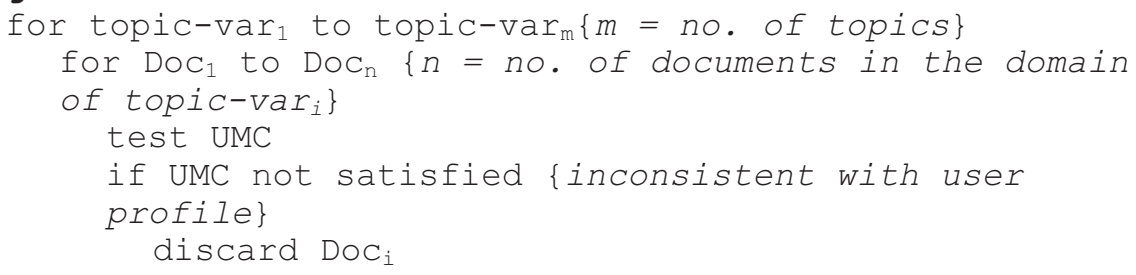

\subsubsection{Co-existence Constraint Satisfaction: Generating the Core Document Set}

Co-existence constraints between two topic-variables need to be satisfied to ensure that their respective selected documents are factually, procedurally and contextually consistent with each other. In practice, co-existence constraints between two topicvariables $_{\mathrm{A \& B}}$ are satisfied if the selected documents from the domain of topic-variable are consistent with selected documents from the domain of topic-variable ${ }_{\mathrm{B}}$. This implies that the document-compatibility conditions (DCC) of a document are not breached by the DCC of another document. A DCC is deemed to be 'breached' when the conflict value (cv), calculated as below, exceeds a predefined threshold.

$$
\begin{gathered}
c v_{D C C^{\prime}}^{D C C^{\prime}}=\mid(\text { weight })_{D C C^{D o c}}^{D o}-(\text { weight })_{D C C^{\prime}}^{D o c^{\prime}} \mid,(\text { type, value })_{D C C}^{D o c}=(\text { type, value })_{D C C^{\prime}}^{D o c} \\
0 \leq c v_{D C C^{\prime}}^{D C C^{\prime}} \leq 1,0 \rightarrow \text { not breached, } 1 \rightarrow \text { breached }
\end{gathered}
$$

To satisfy co-existence constraints, we leverage two CSP search methods: (i) Backtracking (BT) Search; and (ii) Stochastic Generate Test (S-GT) Search. BT search method is used to search the candidate document space to generate the so- 
called core document set-the minimum set of mutually consistent documents such that there exists a single document to cover each topic of interest. BT search incrementally attempts to generate the core document set by (i) choosing an uninstantiated topic-variable, i.e. no document has yet been assigned to the topicvariable; (ii) choosing a candidate document from the domain of the un-instantiated topic-variable; (iii) checking whether the candidate document is consistent with documents that have already been selected to instantiate the other topic-variables; (iv) if the candidate document is consistent-implying that the co-existence constraint is satisfied - the candidate document is selected by instantiating the topic-variable, else the next candidate document within the domain of the same topic-variable is examined. Given that the co-existence constraint cannot be satisfied because all the candidate documents for a topic-variable have been checked, backtracking is performed to select the most recently instantiated topic-variable that may still have some alternative candidate documents. Search proceeds forward again based on the new instantiation of the said topic-variable. Successful BT search ensures that each topic-variable is instantiated with a document, thus resulting in the core document set.

\subsubsection{Co-existence Constraint Satisfaction: Generating the Presentation Set}

Extension of the core document set to the potentially larger presentation document set is performed via two methods: (a) Stochastic Generate and Test (S-GT) and (b) Document Swapping. The motivation for generating the presentation document set is to maximize the information coverage by selecting those candidate documents that do not violate the a priori established factual consistency of the core document set.

The working of the S-GT method is as follows: the non-selected candidate documents are randomly sequenced in $\mathrm{N}$ different sets. Each set is then systematically searched based on the order of the constituent documents in an attempt to include more documents into the core information set without violating the co-existence constraint (i.e. the factual consistency). Consequently, $\mathrm{N}$ presentation document sets are generated, whereby the presentation set with the most documents is selected. We argue that the S-GT method is suitable for this purpose because of its stochastic nature in selecting topic variables and evaluating the documents within their domain in a manner that avoids the 'unfair' effects resulting from a sequenced evaluation of documents as practised by most of the systematic search algorithms.

The application of the earlier CS solving techniques realize factually consistent information set, but the solution is not necessarily optimum-i.e. there may exist a possibility whereby a particular selected document may have caused multiple candidate documents to be discarded during the CSP search. To rectify this limitation, we have developed an information optimization mechanism, termed as document swapping, that 'swaps' a single selected document with multiple discarded candidate documents provided (a) the co-existence constraint is maintained and (b) the presentation document set still contains at least one document for each topic of interest. The outcome of this document-swapping technique is optimized presentation document set, which is the final CSP solution. The document swapping algorithm is given below: 


\section{Algorithm Document Swapping}

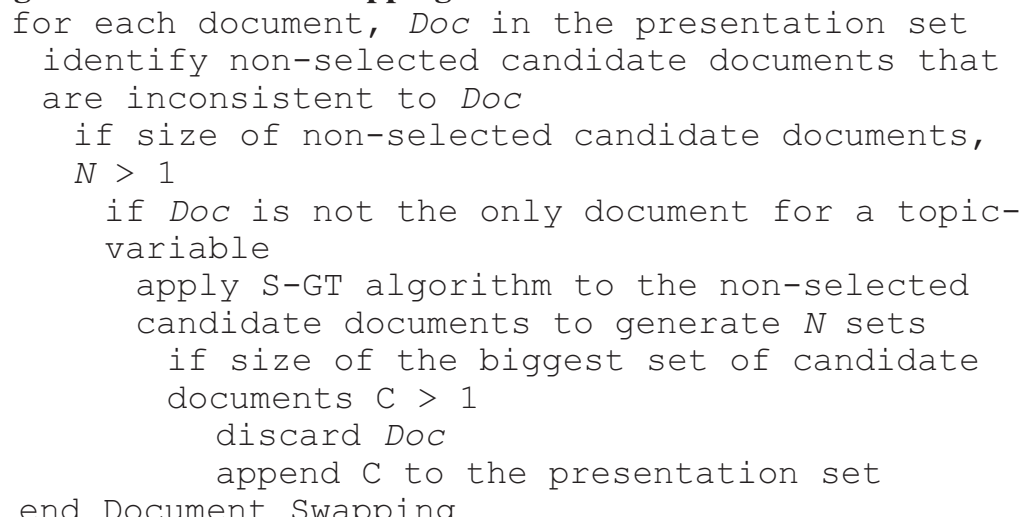

\section{Working Example of Information Customization}

We present a working example of information customization based on the methodology and methods defined above. We are able to partially show the generation of personalized and factually consistent healthcare information.

Step 1 - Generate current user health profile by collecting information from the user's EMR, past profiles and directly from the user. Table 1 shows an exemplar health profile.

Table 1. An exemplar health profile

\begin{tabular}{|c|c|c|}
\hline \multicolumn{2}{|l|}{ Name: $a b c d$} & ID: 5565248 \\
\hline \multicolumn{2}{|l|}{ Health Problem } & Date \\
\hline \multicolumn{2}{|l|}{ Diabetes } & 10 July 2003 \\
\hline \multicolumn{2}{|l|}{ COPD } & 15 August 2003 \\
\hline \multicolumn{3}{|l|}{ User Attributes } \\
\hline Type & Value & Validity \\
\hline Age & 32 & 1 \\
\hline Gender & Female & 1 \\
\hline Blood type & $\mathrm{O}+$ & 1 \\
\hline Family History & Diabetes & 1 \\
\hline Allergy & Pollen & 0 \\
\hline Diet & Vegetarian & 0 \\
\hline Lifestyle & Smoker & 1 \\
\hline Allergy & Seafood & 1 \\
\hline Allergy & Dust & 1 \\
\hline Condition & Pregnant & 0 \\
\hline
\end{tabular}


Step 2- Retrieve topic-specific documents for each health problem, stated in the user's health profile, from the document repository. This gives us the document set shown in Table 2. For CSP purposes, we need to define the topic-variable (topic_var) representing each health problem concerning the user. The domain for each topicvariable comprises the documents that correspond to the topic, shown in Table 3.

Table 2. Topic-specific documents for Diabetes and COPD.

\begin{tabular}{|c|c|c|}
\hline \multirow{2}{*}{ Health Problems } & \multicolumn{2}{|c|}{ Topic-Specific Documents } \\
\hline \multirow{4}{*}{ Diabetes (D) } & Treatment (T) & Medication (M) \\
\cline { 2 - 3 } & DT1 & DM1 \\
\cline { 2 - 3 } & DT2 & DM2 \\
\cline { 2 - 3 } & DT3 & DM3 \\
\cline { 2 - 3 } & DT4 & CM1 \\
\hline \multirow{2}{*}{$\begin{array}{c}\text { COPD - Chronic Obstruc- } \\
\text { tive Pulmonology }(\mathbf{C})\end{array}$} & CT1 & CM2 \\
\cline { 2 - 3 } & CT2 & CM3 \\
\hline
\end{tabular}

Table 3. Definition of variables for topics under diabetes and COPD

$$
\begin{gathered}
\text { Health problem } \\
\text { Topic } \\
\text { Variable :: }\{\text { Domain\} }
\end{gathered}
$$

\section{Diabetes}

Treatment

topic_var1::\{DT1, DT2, DT3, DT4\}

Medication

topic_var2::\{DM1, DM2, DM3, DM4\}

\section{COPD}

Treatment

topic_var3::\{CT1, CT2, CT3, CT4\}

Medication

topic_var4::\{CM1, CM2, CM3, CM4 $\}$

Step 3- Satisfy the user-model constraints to establish the candidate document set. Table 4 shows the result of the node-consistency algorithm on topic_var1, whereby only the relevant documents are selected. 
Table 4. The candidate document set for the health problem diabetes

\begin{tabular}{|c|l|l|c|c|}
\hline Doc & $\begin{array}{c}\text { Document Selection Condi- } \\
\text { tion }\end{array}$ & $\begin{array}{c}\text { Matching User Attribute } \\
\text { from health profile }\end{array}$ & CV & Status \\
\hline DT1 & $<$ family history, diabetes, $1>$ & $<$ family history, diabetes, $1>$ & 0 & Retained \\
\hline DT2 & $<$ lifestyle, smoker, $1>$ & $<$ lifestyle, smoker, $1>$ & 0 & Retained \\
\hline DT3 & $<$ condition, pregnant, $1>$ & $<$ condition, pregnant, $0>$ & 1 & Discarded \\
\hline DT4 & $<$ allergy, seafood, $1>$ & $<$ allergy, seafood, $1>$ & 0 & Retained \\
\hline
\end{tabular}

Step 4- Satisfy the co-existence constraints to generate the core document set and presentation document set. Given the document co-existence conditions for each document (which cannot be shown due to lack of space), Table 5 shows the core set. Next, we apply the S-GT algorithm to maximize the information coverage. Table 6 shows the three random sets of non-selected documents, whereby the second random set best maximizes the information coverage. This is because CT4, which is inconsistent with both DM4 and DT4, was positioned only after DM4 in the random set. Such ordering of documents enabled DM4 to be selected first instead of CT4 and thus blocked CT4 to be selected subsequently. Without CT4 in the presentation set, DT4 can be next selected. Note that this is not possible for the other two random sets.

Table 5. Core document set resulting from BT search

\begin{tabular}{|l|l|c|c|c|}
\hline Topic-variables & \multicolumn{1}{|c|}{$\begin{array}{c}\text { Domain (Candi- } \\
\text { date set) }\end{array}$} & $\begin{array}{c}\text { Selected } \\
\text { (Core set) }\end{array}$ & Discarded & $\begin{array}{c}\text { None- } \\
\text { selected }\end{array}$ \\
\hline Topic_var1 & DT1, DT2, DT4 & DT1 & - & DT2, DT4 \\
\hline Topic_var2 & DM1, DM3, DM4 & DM1 & - & DM3, DM4 \\
\hline Topic_var3 & $\begin{array}{l}\text { CT1, CT2, CT3, } \\
\text { CT4 }\end{array}$ & CT3 & CT1, CT2 & CT4 \\
\hline Topic_var4 & CM1, CM2, CM3 & CM1 & - & CM2 \\
\hline
\end{tabular}

Table 6. An extended presentation document set resulting from S-GT search

\begin{tabular}{|l|l|c|c|}
\hline Non-selected (Randomised order) & \multicolumn{1}{|c|}{ Documents selected } & $\begin{array}{c}\text { Documents } \\
\text { discarded }\end{array}$ & Size \\
\hline CT4, DT4, DM3, DM4, DT2, CM2 & CT4, DM3, DT2 & DT4, DM4, CM2 & 3 \\
\hline DM4, CT4, DT4, DM3, CM2, DT2 & DM4, DT4, DM3, DT2 & CT4, CM2 & $\mathbf{4}$ \\
\hline CM2, CT4, DM4, DT2, DM3, DT4 & CT4, DT2, DM3 & CM2, DM4, DT4 & 3 \\
\hline
\end{tabular}

Step 5- Apply document swapping to optimize the presentation document set. Table 7 shows the presentation document set (comprising 8 documents) together with their conflicts with the non-selected documents. DT1 has been detected to be block the inclusion of CT1 and CM2. Via document swapping, DT1 can be discarded to select CT1 and CM2, without disturbing the factual consistency, thereby increasing the size of the (optimized) presentation document (as shown in Table 8). The resultant optimized presentation document set is the personalized and factually consistent information that need to be presented to the user. 
Table 7. Presentation document set before optimization

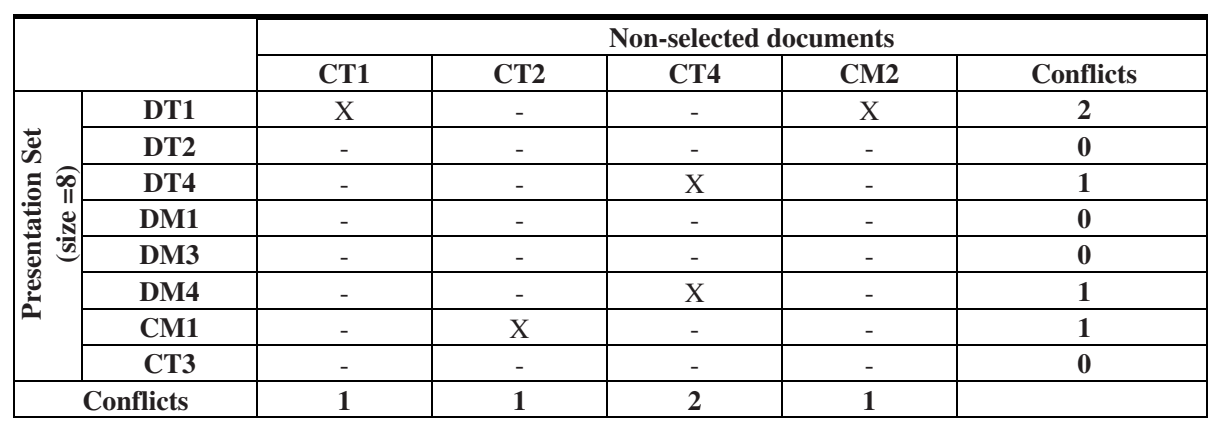

Table 8. Presentation document set after optimization

\begin{tabular}{|c|c|c|c|c|c|}
\hline & & \multicolumn{3}{|c|}{ Non-selected docs } & \multirow{2}{*}{ Conflicts } \\
\hline & & CT2 & CT4 & DT1 & \\
\hline \multirow{9}{*}{ 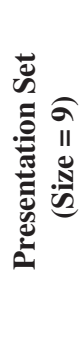 } & DT2 & - & - & - & $\mathbf{0}$ \\
\hline & DT4 & - & $X$ & - & 1 \\
\hline & DM1 & - & - & - & 0 \\
\hline & DM3 & - & - & - & $\mathbf{0}$ \\
\hline & DM4 & - & $X$ & - & 1 \\
\hline & CM1 & $\mathrm{X}$ & - & - & 1 \\
\hline & CT3 & - & - & - & $\mathbf{0}$ \\
\hline & CT1 & - & - & X & 1 \\
\hline & CM2 & - & - & $\mathrm{X}$ & 1 \\
\hline \multicolumn{2}{|c|}{ Conflicts } & 1 & 2 & 2 & \\
\hline
\end{tabular}

Step 6- Prepare the final information package based on the optimized presentation document set. The XML based documents are transformed to a pre-defined HTML template to realize an HTML based, including text and images, information package.

Step 7- Deliver the information package to the user via Internet mediated mechanisms. A push based mechanism is implemented to send the customized information package to the user via email.

\section{Concluding Remarks}

In this paper we presented and demonstrated a services-oriented information customization framework that leverages on a unique hybrid of adaptive hypermedia and constraint satisfaction methods. The work has direct implication towards efforts to retain a stable client-base for E-services via a web portal. It is routinely suggested that enterprises need to understand their customers in terms of their current needs, preferences and future goals. One way of manifesting this understanding of customer needs 
is to pro-actively customize services/information content, leading to improved customer relationship management (CRM). In fact, according to Brohman et al "CRM solutions are deemed to be so critical that investments in them continue to be funded, even in these days of shriveling IT budgets" [26]. We believe that our work, contributes to this end by presenting an information customization framework that provides adaptive personalized information content through a static user-model. Most importantly, the incorporation of constraint satisfaction methods ensure that not only the information content is consistent with the user-model but it is also factually, procedurally, and contextually consistent. Indeed, for effective CRM it is imperative to both account for and satisfy these qualitative constraints.

We conclude that person-specific customization of information viz. a user-model is a complex task that necessitates a systematic, pragmatic and multifaceted methodology. Previously, knowledge-based reasoning, case-based reasoning, natural language processing and other artificial intelligence methods have been successfully applied for user modeling and information personalization. In this paper, we have demonstrated the novel application of constraint satisfaction methods for information customization, which offers an interesting synergy between adaptive hypermedia and constraint satisfaction methods. From our experiments, we have concluded that (a) by varying the constraints the degree of information personalization can be modulated with interesting outcomes; and (b) apart from text-based hypermedia documents, other hypermedia objects such as picture, movie or sound-based documents can be used within the final information package.

Information personalization in an E-Healthcare context for the purposes for patient education and empowerment is quite prevalent $[8,27,28]$ and there is a growing demand for adaptive websites that deliver personalized wellness maintenance information on an individual and community basis. We have applied our IC framework towards the dissemination of personalized healthcare information, for a limited number of medical problems, via a restricted website managed by medical professionals.

In conclusion, we believe that this is the first step towards the incorporation of constraint satisfaction within an IC paradigm. Also, the realization to ensure factual consistency when amalgamating heterogeneous information will lead to interesting research in adaptive service-oriented systems. Finally, we believe that the featured IC approach can be used for a variety of E-services for education material customization, stock market reporting and advice, tourist information and so on; the only limitation is the specification of co-existence constraints.

\section{References}

[1] Rust RT, Lemon KN, E-service and the consumer. International Journal of Electronic Commerce, Vol. 5(3), 2001 pp. 85-102.

[2] Helal S, Su S, meng J, Krithivasan R, Jagatheesan A, The Internet enterprise. Proceedings of Symposium on Application and the Internet, 2002. 
[3] Zirpins C, Weinreich H, Bartelt A, Lamersdorf W, Advanced concepts for next generation portals. Proceedings of 12th International Workshop on Database and Expert Systems Applications, 3-7 Sept. 2001, pp. 501-506.

[4] Piccinelli G, Salle M, Zirpins C, Service-oriented modelling for e-business applications components. Proceedings of $10^{\text {th }}$ IEEE International Workshops on Enabling Technologies: Infrastructure for Collaborative Enterprises (WET ICE), 20-22 June 2001, pp. 12-17.

[5] Bogonikolos N, Makris C, Tsakalidis A, Vassiliadis B, Adapting information presentation and retrieval through user modeling. Proceedings of International Conference on Information Technology: Coding and Computing, 2-4 April 2001, pp. 399-404.

[6] Zhang Y, Im I, Recommender systems: A framework and research issues. Proceedings of American Conference on Information Systems, 2002.

[7] Fink J, Kobsa A, Putting personalization into practice, Communications of the ACM, Vol. 45(5), 2002.

[8] Abidi SSR, Chong Y, Abidi SR, Patient empowerment via 'pushed' delivery of personalized healthcare Educational content over the internet. Proceedings of $10^{\text {th }}$ World Congress on Medical Informatics, 2001, London.

[9] Brusilovsky P, Methods and techniques of adaptive hypermedia. In P. Brusilovsky, A. Kobsa and J. Vassileva (eds.): Adaptive Hypertext and Hypermedia. Kluwer Academic Publishers, Dordrecht, 1998a, pp. 1-43.

[10] Brusilovsky P, Kobsa A, Vassileva J (Eds.), Adaptive Hypertext and Hypermedia. Kluwer Academic Publishers, Dordrecht, 1998b.

[11] Kobsa A, Personalized hypermedia presentation techniques for improving online customer relationships. The Knowledge Engineering Review, Vol. 16(2), 1999, pp. 111155.

[12] Perkowitz, M, Etzioni O, Adaptive web sites. Communications of the ACM, Vol. 43(8), 2000, pp. 152-158.

[13] Henze N, Nejdl W, Extendible adaptive hypermedia courseware: integrating different courses and web material. In P. Brusilovsky, O Stock \& C Strappavara (Eds.) Adaptive Hypermedia and Adaptive Web-based Systems, Springer Verlag, Berlin, 2000, pp. 109120.

[14] Kobsa A, Muller D, Nill A, KN-AHS: an adaptive hypermedia client of the user modeling system BGP-MS. Proceeding of the Fourth International Conference on User Modeling, 1994, pp. 99-105.

[15] Boyle C, Encarnacion AO, MetaDoc: An adaptive hypertext reading system. User Models and User Adapted Interaction, Vol. 4(1), 1994, pp. 1-19.

[16] Ardissono L, Goy A, Tailoring the interaction with users in web stores, User Modelling and User Adapted Interaction, Vol. 10(4), 2000, pp. 251-303.

[17] Hohl H, Bocker HD, Gunzenhauser R, HYPADAPTER: an adaptive hypertext system for exploratory learning and programming, User Modelling and User Adapted Interaction, Vol. 6(2), pp. 131-155.

[18] Kaplan C, Fenwick J, Chen J, Adaptive hypertext navigation based on user goals and context, User Modelling and User Adapted Interaction, Vol. 3(3), pp. 193-220.

[19] Oppermann R, Specht M, A context sensitive nomadic information system as an exhibition guide, Proceedings of the Handheld and Ubiquitious Computing Second International Symposium (HUC 2000), 2000, pp. 127-142.

[20] Tsang E, Foundations of constraint satisfaction. Academic Press, London, UK. 1993. 
[21] Sabin D, Freuder E, Configuration as composite constraint satisfaction. Proceedings of the Artificial Intelligence and Manufacturing Research Planning Workshop, 1996, pp.153-161.

[22] Torrens M, Faltings B, SmartClients: Constraint satisfaction as a paradigm for scaleable intelligent information systems. Workshop on Artificial Intelligence on Electronic Commerce, AAAI-99, 1999, Orlando, Florida, USA.

[23] De Rosis F, Pizzutilo S, Russo A, User tailored hypermedia explanations. INTERCHI'93 Adjunct proceedings, Amsterdam, 1993.

[24] Beaumont IH, User modeling in the interactive anatomy tutoring system ANATOMTUTOR. User Models and User Adapted Interaction, Vol. 4(1), 1994, pp. 21-45.

[25] Barták R, Constraint programming: In pursuit of the holy grail. Proceedings of the Week of Doctoral Students (WDS99), Part IV, MatFyzPress, Prague, 1999, pp. 555564.

[26] Brohman MT, Watson RT, Piccoli G, Parasuraman A, Data completeness: A key to effective net-based customer service systems. Communications of the ACM, Vol. 46(6), 2003, pp. 47-51.

[27] Bental D, Adapting web-based information to the needs of patients with cancer. Proceedings of International Conference On Adaptive Hypertext and Adaptive Web-based Systems, Trento, Italy, 2000, pp. 27-37.

[28] Wilke W, Bergmann R, Techniques and Knowledge Used for Adaptation During Case Based Problem Solving. Lecture Notes in Artificial Intelligence, Vol. 1416. SpringerVerlag, Berlin, 1998. 